\title{
ANFOTERICINAB DEOXICOLATO: EXPERIENCIA TERAPEUTICA EN CRIPTOCOCOSIS MENINGEA EN PACIENTES VIH ADULTOS DEL HOSPITAL CARLOVAN BUREN
}

\author{
(Anphotericin B deoxicolate, therapeutic experience in meningeal \\ cryptococcosis in a VIH adult patients of Carlos Van Buren Hospital)
}

\author{
Pablo Herrera (1), Rodrigo Cruz Ch (2) \\ 1. Interno de Medicina Universidad de Valparaíso \\ 2. Cátedra de Micología Universidad deValparaíso, \\ UCI Adultos Hospital San Felipe.
}

Palabres clave: Anfotericina $\mathrm{B}$, criptococosis meníngea, $\mathrm{VIH}$, experiencia terapéutica

Key words:Anphotericin B, meningeal cryptococcosis, VIH, therapeutic experience

\section{RESUMEN}

La anfotericina $B$ es un antifungico ampliamente usado en infecciones sistémicas por hongos levaduriformes y filamentosos, entre ellas las meningitis por Cryptococcus neoformans. Sus reacciones adversas pueden ser inmediatas o dosis y tiempo dependiente. En nuestro trabajo en el hospital C.van Buren de Valparaíso, se revisaron 27 fichas de pacientes que cumplieron con los criterios de inclusión (24 hombres y 3 mujeres). El principal sintoma de la meningitis por Crytococcus fue la cefalea (96,3\%). En 25 casos hubo confirmación con tinta china y/o cultivo. Durante el tratamiento con anfotericina $B$ la hipokalemia fue la reacción adversa que se presento con mayor frecuencia (83\%) y la nefrotoxicidad en un 59,1\%. La dosis acumulada administrada fue en promedio $525 \mathrm{mg}$, suspendiendo generalmente su administración cuando se lograba una mejoría clínica junto a esterilidad del LCR (tinta china y/o cultivo negativo). Un 33,3\% de los pacientes con diagnostico de meningitis por C. neoformans falleció por distintas complicaciones.

\section{INTRODUCCION}

La criptococosis es una infección sistémica causada por el hongo levaduriforme

Cryptococcus neoformans, un saprófito ubicuo en la naturaleza, sin un área endémica definida.Algunos de los distintos serótipos del hongo (A y D) crecen principalmente en las heces de

Recibido el 4 de Noviembre 2009

Aceptado el 29 de Diciembre 2009

\section{ABSTRACT}

Amphotericin B is a antifungal drug widely used in systemic infections by filamentous and levaduriform fungi, as in meningitis by Cryptococcus neoformans. Its adverse reactions can be immediate or dose and time dependent. In our paper, 27 patient files wer e reviewed in C.van Biur en hospital of $V$ alparaiso and all of them met the criteria for inclusion (24 men and 3 women). In 96.3\% of cases, headaches were the main symptom of meningitis caused by Cryptococcus. Twenty five cases were confirmed by the use of chinese ink and/or by culture. During the treatment with amphotericin B, the most frequent changes were hypokalemia (83\%) and nefrotoxicity in $59.1 \%$. The average of cumulative dose administered was $525 \mathrm{mg}$ and was suspended when a clinical recovery was achieved next to a sterile cefalorraquid liquid (negative to chinese ink and/or culture). $33.3 \%$ of the patients diagnosed with meningitis by $\boldsymbol{C}$. neoformans died due to different complications.

palomas y otras aves sin que ellas se vean infectadas, así como en otras fuentes en la naturaleza. La criptococosis ocurre tanto en animales como en seres humanos, siendo la vía respiratoria la principal puerta de entrada. (Quintero et al.,2005 ;Castelláet al.,2008)

El SIDA es el factor de riesgo mas importante, sin embargo, esta enfermedad también puede presentarse en pacientes transplantados y en aquellos con procesos malignos linforreticulares (Sepulveda \&Afani, 2002). Clínicamente puede cursar con síntomas leves e 
inespecíficos, como cefalea, nauseas, mareos, irritabilidad, somnolencia, confusión, obnubilación o conductas bizarras. Cuando se afectan los nervios craneales se presenta menor agudeza visual, diplopía o paresia.

Las convulsiones en general solo se presentan en una fase avanzada de la enfermedad. Los pacientes a menudo se presentan afebriles y en la mayor parte de los casos la rigidez de nuca es mínima o casi nula. (Reese \& Betts, 2004).

La forma de diagnosticar una criptococosis meningea es mediante la prueba de tinta china en LCR (rendimiento 40 a $50 \%$ ), cultivos en LCR y/o detección de antígeno polisacárido capsular en LCR. (Fica, 2004; Reese $\&$ Betts, 2004).

El tratamiento de la criptococosimneníngea se basa principalmente enAnfotericina B deoxicolato $0,7 \mathrm{a} 1 \mathrm{mg} /$ $\mathrm{kg}$ /día por 15 a 20 días (dosis acumulada total de $500-$ $1000 \mathrm{mg}$ ), si existe esterilización de LCR asociado a una franca mejoría clínica, se puede continuar con fluconazol $400 \mathrm{mg} /$ día por 8 a 10 semanas. De forma profiláctica los pacientes se deben mantener con fluconazol $200 \mathrm{mg}$ /día hasta que su recuento de CD4 se eleve sobre 100-200/mm por más de 6 meses. (Gilbertet al., 2009; Burke,2008)

\section{Anfotericina B Deoxicolato.}

Desde que en 1956, Gold y colaboradores aislaron la AnfotericinaB (AmB) desde Streptomyces nodosus, este fármaco se ha constituido en el tratamiento de elección para las micosis sistémicas como Aspergilosis, Blastomicosis, Candidosis, Coccidiodomicosis, Criptococosis, Cromomicosis, Esporotricosis, Histoplasmosis y Mucormicosis (Hardman et al., 2003; Mandellet al., 2002).

Se caracteriza por ser insoluble en agua, por ello se asocia de forma tradicional a deoxicolato, una sal biliar con la que forma un coloide con el agua.

El mecanismo de acción de la AmB, se basa en la unión del fármaco al ergosterol de la membrana celular del hongo, generando la formación de canales que facilitan la salida del contenido citoplasmico y la consecuente muerte celular. (Laniado-Laborín \& Cabrales-\$rgas, 2009).

Por vía oral solo se absorbe un $5 \%$, por lo que se debe administrar de forma endovenosa. La adición de electrolitos a la solución de venoclisis produce precipitación del coloide, es por ello, que se recomienda su infusión con solución glucosada al 5\%. En plasma la anfotericina B es separada de su complejo con deoxicolato, uniéndose ampliamente a lipoproteínas $(91-95 \%)$, a los eritrocitos, el colesterol y membranas de los distintos tejidos del cuerpo. Su concentración máxima depende de la dosis, la frecuencia y velocidad de infusión. En adultos una infusión EV de $0.6 \mathrm{mg} / \mathrm{kg}$ alcanza un Peak plasmatico de $1-3 \mathrm{mg} / \mathrm{L}$, que decae luego a una fase estable prolongada de $0.2-0.5$ $\mathrm{mg} / \mathrm{L}$. En liquido peritoneal, pleural y sinovial las concen- traciones de anfotericina $\mathrm{B}$ son menores del $50 \%$ respecto al plasma, mientras que en LCR no llegan al $10 \%$. Se metaboliza parcialmente en el hígado y se elimina por la bilis y la orina en escasa proporción (5-10\%), por lo que no es necesario modificar sus dosis en insuficiencia hepática, renal o hemodiálisis. (Flórez \&Armijo, 2003). Muchas veces los efectos adversos derivados de las terapias con $\mathrm{AmB}$, son los que limitan su administración. En este sentido se pueden considerar dos tipos de reacciones adversas :

a) Inmediatas: concomitantes a la administración endovenosa; escalofríos, fiebre, cefalea, vómitos e hipotensión leve. La interleuquina-1 y el factor de necrosis tumoral de monocitos y macrófagos estarían vinculados con estas respuestas (rara vez ocurren reacciones anafilácticas). La administración rápida, en menos de 60 min, puede generar arritmias y paro cardíaco, por lo que se recomienda administrar en un lapso de $3-6$ hrs. (LaniadoLaborín \& Cabrales- brgas, 2009).

b) Dosis y tiempo dependientes: durante el tratamiento, existe un alto riesgo de nefrotoxicidad, es el efecto adverso más importante y revierte con el cese de la terapia en la mayoría de los casos; el proceso se asocia con una disminución del filtrado glomerular y el flujo sanguíne o renal, así como fallas en la reabsorción de electrolitos en los túbulos proximales y distales. Clínicamente se manifiesta por aumento de la creatinina plasmática y del nitrógeno uréico. Asimismo, en la medida que aumentan las cagas y días de terapia, se puede observar anemia, trombocitopenia, leucopenia, hipokalemia, hiponatremia, acidosis y tromboflebitis. (Bagnis \& Deray, 2002 ; Berdichevski et al., 2006).

Se han intentado distintas estrategias para disminuir la toxicidad de laAmB, como por ejemplo administrar $1 \mathrm{mg}$ en $20 \mathrm{ml}$ de suero glucosado al 5\%, como dosis de prueba para intentar predecir la susceptibilidad del paciente al fármaco o la administración de $1 \mathrm{~L}$ de solución salina previo a la terapia para reducir la nefrotoxidad.

Actualmente existen las formulaciones lipidicas de la anfotericina B, destacando entre ellas laAnfotericina B liposomal (AmBisome ${ }^{\circledR}$ ), que incluye el principio activo en liposomas pequeños de una sola capa lipidica $<0.15 \mu \mathrm{m}$ de diámetro. Con estas formulaciónes se reducen los efectos tóxicos del fármaco (principalmente la nefotoxicidad) conservando la efectividad del antifúngico (Thompson, 1997; Walsh et al., 1999; Cruz , 2004).

La realidad hospitalaria en la que nos desenvolvemos no siempre posibilita disponer de nuevos tratamientos, por lo que es necesario conocer la dosificación de las ,drogas con las que contamos, sus efectos adversos y la efectividad en cada infección.

Los objetivos planteados en este trabajo fu eron: 
determinar las características clínicas de los pacientes VIHSIDA con meningitis por $\boldsymbol{C}$. neoformans, además de otorgar una imagen del uso de la $\mathrm{AmB}$ deoxicolato en el Hospital Carlos van Buren, enfatizando en las dosis acumuladas, los días de tratamiento, las reacciones adversas y principales causas de suspen sión.

\section{MATERIALES YMETODOS}

Se efectuó un análisis descriptivo retrospectivo mediante el estudio de las historias clínicas de los pacientes con meningitis por $C$. neoformans tratados con Am B deoxicolato, entre Enero del 2000 y Diciembre del 2008, en el hospital Carlos van Buren deValparaíso. Para ello se solicitó la lista de pacientes, de acuerdo al código del fármaco, al departamento de informática de este recinto hospitalario. Del listado total se seleccionaron las fichas clínicas que cumplían con:

Criterios de inclusión: a) Paciente mayor de 18 años, b) Diagnostico de VIH antes o durante hospitalización, c) Diagnostico de criptotocosis meníngea mediante tinta china, cultivo de hongos o serología.

Se efectuó un análisis de los exámenes de laboratorio y la evolución clínica en el periodo de diagnóstico y tratamiento de la criptococosis

Definición de fiebre temperatura corporal $37,5^{\circ} \mathrm{C}$.

Definición de hipokalemia: concentración plasmatica de $\mathrm{K}^{+}<3.5 \mathrm{mmol} / \mathrm{L}$.

Definición de nefrotoxicidad decrecimiento de un $50 \%$ o más del clearance de creatinina o un incremento de $0,5 \mathrm{mg} /$ $\mathrm{dl}$ de la creatininemia desde el nivel basal.

Definición de leucopenia : disminución de leucocitos totales bajo $4.3 \times 10 / \mathrm{L}$.

Definición de prueba de sensibilidad a hnfotericina $B$ administración de $1 \mathrm{mg}$ de $\mathrm{AmB}$ deoxicolato en $20 \mathrm{ml}$ de suero glucosado al $5 \%$, antes de iniciar tratamiento antimicótico.

Los datos se tabularon y analizaion en el programa estadístico SPSS 15.0.

\section{RESULTADOS}

Del total de fichas clínicas revisadas, 27 cumplieron con los criterios de inclusión en el trabajo. Del total de pacientes, $24(88,9 \%)$ son hombres y $3(11,1 \%)$ mujeres. La media de edad fue de 37 años con un rango de $23-58$.

Al momento de la hospitalización 14 (51,86\%) pacientes no habían sido diagnosticado como VIH, $13(48,14 \%)$ eran VIH positivos; de ellos $6(46,2 \%)$ se encontraban en tratamiento retroviral, el cual se había iniciado entre 7 y 60 días previos al ingreso.
Tabla 1. Manifestaciones clínicas al momento de la hospitalización.

\begin{tabular}{|l|c|c|}
\hline \multicolumn{1}{|c|}{ Síntomas } & $\begin{array}{c}\mathbf{N}^{\mathbf{2}} \\
\text { pacientes }\end{array}$ & $\begin{array}{c}\text { Porcentaje del } \\
\text { total (n=27) }\end{array}$ \\
\hline Cefalea & 26 & $96,3 \%$ \\
Fiebre & 14 & $51,9 \%$ \\
Candidosis & 13 & $48,1 \%$ \\
Comp. de conciencia & 12 & $44,4 \%$ \\
Signos meníngeos & 4 & $14,8 \%$ \\
Parálisis ocular & 2 & $7,4 \%$ \\
\hline
\end{tabular}

La mayoría de los pacientes debutó clínicamente con cefalea $(96,3 \%)$, menos frecuente fiebre, candidosis y compromiso de conciencia. Los signos meníngeos y la parálisis ocular solo fueron descritos en una minoría. (Tabla 1). Del total de pacientes, 16 (59,3\%) presentaron leucopenia y $11(40,7 \%)$ nola presentaron.

El diagnostico de criptococosis meníngea que motivó el inicio del tratamiento, se efectuó principalmente mediante el resultado de tinta china $(+)$ del estudio del LCR en 19 pacientes $(70,4 \%)$, en menor medida se utilizó el cultivo de hongos en 4 pacientes (Tabla 2).

Tabla 2. Examen clínico del LCR que se utilizó para diagnóstico e inicio de tratamiento

\begin{tabular}{|l|c|c|}
\hline \multicolumn{1}{|c|}{ Examen Diagnóstico } & $\begin{array}{c}\mathrm{N}^{\mathrm{o}} \\
\text { Pacientes }\end{array}$ & $\begin{array}{c}\% \text { del } \\
\text { Total }\end{array}$ \\
\hline Tinta china & 19 & $70,4 \%$ \\
Cultivo & 4 & $14,8 \%$ \\
Tinta china y cultivo & 2 & $7,4 \%$ \\
Clínica & 2 & $7,4 \%$ \\
Serología & 0 &, $0 \%$ \\
Total & 27 & $100,0 \%$ \\
\hline
\end{tabular}

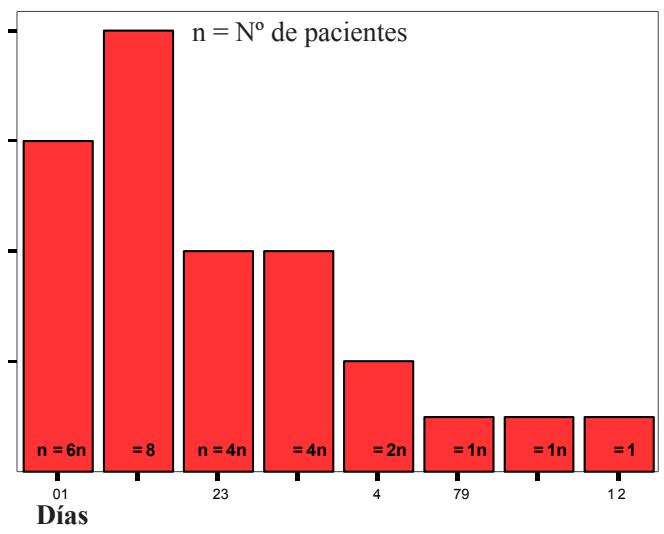

Figura 1. Inicio de tratamiento con anfotericina $B$ (días) desde el diagnóstico de criptococosis meníngea 
Desde el momento del resultado del examen al inicio del tratamiento con $\mathrm{AmB}$, en 22 casos $(82,4 \%)$ transcurrieron menos de $72 \mathrm{hrs}$. El resto de los pacientes se trataron en un lapso de hasta 12 días. (Figura 1).

En los pacientes en los que se decidió iniciar tratamiento antimicótico, se efectuó prueba de sensibilidad a la Anfotericina en 15 de ellos $(55,6 \%)$. El tiempo de tratamiento fluctuó entre 7 y 60 días con una mediana de 22. La dosis acumulada de AmB, presentó una variación entre 100 y $1760 \mathrm{mg}$, con una mediana de $525 \mathrm{mg}$.

En 24 pacientes que mantenían tratamiento con AmB, se controló ELP, de ellos 20 (83,3\%) presentaron hipokalemia. La instalación de ésta fue en promedio de 6 días con una fluctuación entre 1 y 14 días, la corrección de la alteración se efectuó en 5 días en promedio, con una variación entre 1 y 22 días.

En 22 pacientes se controló la función renal, de ellos 13 (59,1\%) desarrollaron nefrotoxicidad. La instauración fue en promedio de 10 días (Tabla 3) .

Tabla 3. Tiempo (días) de instauración de nefrotoxicidad en pacientes con criptococosis en tratamiento conAnfotericina $B$.

\begin{tabular}{|c|c|c|}
\hline \multicolumn{3}{|c|}{ INSTAURACION DE } \\
NEFROTOXICIDAD (días) \\
\hline Mediana & Mínimo & Máximo \\
\hline 10 & 4 & 18 \\
\hline
\end{tabular}

Si bien no se estableció una relación estadísticamente significativa entre la presencia de nefrotoxicidad e hipokalemia, se observa más hipokalemia (13 casos) en los pacientes que presentan nefrotoxicidad respecto a los que no ( 7 casos).

Independiente del fallecimiento del paciente, en 22 casos se suspendió la administración de AmB, de ellos la principal causa fué la negativización micológica del LCR y la buena respuesta clínica al tratamiento (Figura 2). En

Tabla 4. Complicaciones médicas en el curso del tratamiento conAnfotericina $B$ en pacientes con criptococosis.

\begin{tabular}{|l|c|c|}
\hline \multicolumn{1}{|c|}{ Complicación } & $\begin{array}{c}\mathrm{N}^{\mathrm{o}} \\
\text { pacientes }\end{array}$ & $\begin{array}{c}\% \text { del total } \\
(\mathrm{n}=27)\end{array}$ \\
\hline Neumonía aspirativa & 4 & 14,8 \\
Agitación psicomotora & 3 & 11,1 \\
Reacción alérgica & 2 & 7,4 \\
Sepsis por CVC & 2 & 7,4 \\
HDA & 2 & 7,4 \\
HSA & 1 & 3,7 \\
Neuritis óptica & 1 & 3,7 \\
Absceso cerebral & 1 & 3,7 \\
Neumotórax & 1 & 3,7 \\
\hline
\end{tabular}

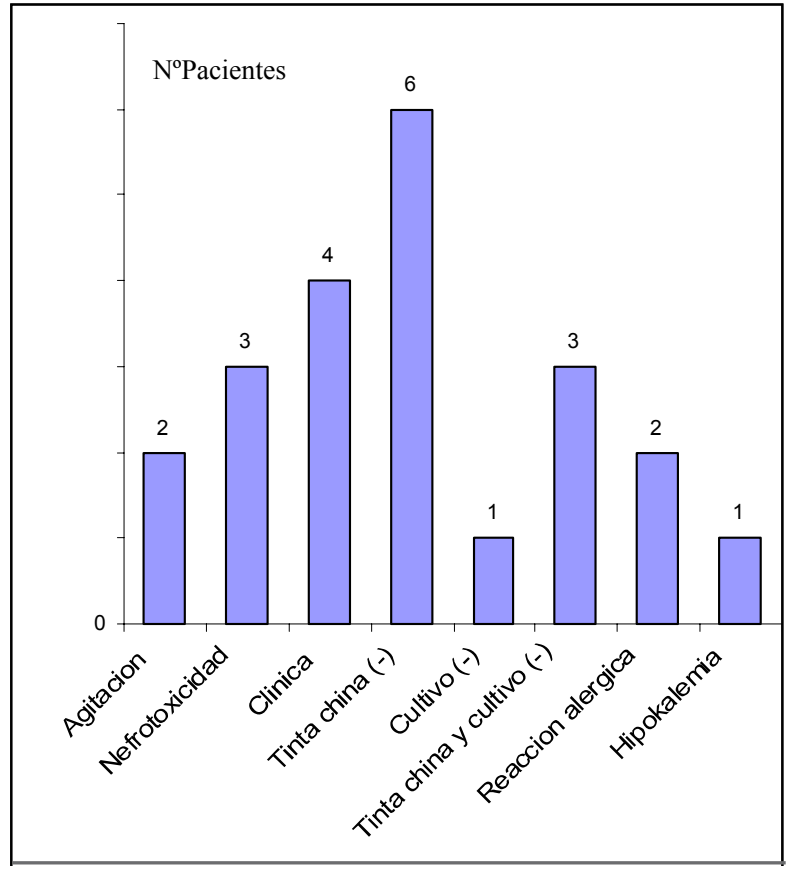

Figura 2. Causa de término de tratamiento con Anfotericina B en pacientes con criptococosis.

quienes se suspendió el tratamiento por reacción alérgica, nefrotoxicidad e hipokalemia ( 6 casos), se efectuó prueba de sensibilidad a la AmB en 3 de los casos.

Aparte de nefrotoxicidad e hipokalemia, los pacientes con criptococosis que se encontraban en tratamiento con $\mathrm{AmB}$, presentaron como complicación médica: neumonía aspirativa y agitación psicomotora más frecuentemente (Tabla 4).

Del total de pacientes tratados, $9(33,3 \%)$ fallecieron, de ellos la principal causa de muerte fue FOM en 7 casos y falla respiratoria en 2Todos quienes desarrollaron complicaciones pulmonares (neumonía aspirativa y neumotorax) fallecieron. En relación a la dosis acumulada de AmB administrada a cada paciente, $13(48,2 \%)$ pacientes recibieron menos de $500 \mathrm{mg}$ de tratamiento, 7 de ellos fallecieron, representando al 77,8\% del total (Tabla 5)

Tabla 5. Dosis acumulada dAnfotericina $B$ administrada y numero de fallecidos por grupo.

\begin{tabular}{|c|c|c|c|c|}
\hline $\begin{array}{c}\text { Dosis } \\
\text { Acumulada } \\
(\mathrm{mg})\end{array}$ & $\begin{array}{c}\mathrm{N}^{\mathbf{0}} \\
\text { pacientes }\end{array}$ & $\begin{array}{c}\% \\
\text { del total } \\
(\mathrm{n}=27)\end{array}$ & $\begin{array}{c}\mathrm{N}^{\mathbf{0}} \\
\text { fallecidos }\end{array}$ & $\begin{array}{c}\% \text { del total de } \\
\text { fallecidos } \\
(\mathrm{n}=9)\end{array}$ \\
\hline$<500$ & 13 & 48,2 & 7 & 77,8 \\
$500-1000$ & 11 & 40,7 & 1 & 11,1 \\
$>1000$ & 3 & 11,1 & 1 & 11,1 \\
& & & & \\
TOTAL & 27 & 100,0 & 9 & 100,0 \\
\hline
\end{tabular}




\section{DISCUSION}

La Criptococosis meníngea en el grupo estudiado, se presentó como enfermedad marcadora en los pacientes sin diagnostico de $\mathrm{VIH}$, especialmente como complicación en individuos VIH positivos que no tenían tratamiento antiretroviral (T AR) o en el contexto de síndrome de reconstitución auntoinmune en quienes habían iniciado TAR hace menos de 60 días. Los pacientes en su mayoría fueron hombres jóvenes en promedio de 37 años.

Debido a la situación de inmunosupresion en la que se presenta la infección porC. neoformans, la clínica es poco florida, a excepción de la cefalea. Otros síntomas o signos clínicos sólo se expresan en la mitad o menos de los pacientes. En el contexto de un paciente con cefalea persistente, el análisis de laboratorio del LCR con tinta china, es una buena prueba para establecer el diagnóstico e iniciar el tratamiento. Al respecto, y considerando las limitaciones de la tinta china para el diagnóstico de esta micosis, su resultado debe ser interpretado con criterio y de acuerdo al contexto clínico, complementándolo con otras técnicas de identificación, tales como el cultivo para hongos y la prueba de aglutinación del látex, de manera de evitar falsos negativos. En nuestro estudio la mayoría de las meningitis fue diagnosticada por tinta china, no utilizándose la reacción de látex, técnica con una mayor sensibilidad y una alta especificidad en el diagnostico de esta enfermedad (Reese \& Betts, 2004). En 2 casos se diagnosticó solo por clínica, situación difícil, ya que no existen síntomas específicos de meningitis por

Criptococcus. En nuestros pacientes sólo la cefalea se presentó en la mayoría, síntoma por lo demás común a otros agentes y a otras etiologías.

Durante el tratamiento conAmB, la hipokalemia fue la alteración que se presentó con mayor frecuencia, junto a la nefrotoxicidad (cercana al 60\%), cuya prevalencia es concordante con lo reportado por varios investigadores (Luke, 1998; Nucci et al., 1999; Bagnis \& Deray, 2002; Laniado-Laborín \& Cabrales-Vargas 2009). Asimismo, pese a que no se estableció una correlación estadísticamente significativa, existe una tendencia de más hipokalemia en la medida que exista nefrotoxicidad. Por esto, es indispensable el control de de ELP y función renal en todos quienes reciben tratamiento con AmB. De un total de 27 pacientes analizados, en 24 y 22 se controló kalemia y creatininemia respectivamente, situación preocupante, pues no debería quedar ningún paciente sin estos controles seriados. Como complicación durante el tratamiento, destaca por su frecuencia la neumonía aspirativa, la que se debe probablemente al compromiso de conciencia secundario a la meningitis que pueden presentar estos pacientes.

El tratamiento con anfotericina $\mathrm{B}$ deoxicolato se inicio en la mayoría de los casos antes de las $72 \mathrm{~h}$ desde el diagnóstico de criptococosis, lo que disminuye el riesgo de complicaciones y de mortalidad.

La dosis acumulada administrada fue en promedio $525 \mathrm{mg}$, suspendiendo generalmente su administración cuando se lograba una mejoría clínica junto a esterilidad del LCR (tinta china y/o cultivo negativo). Con este esquema, un $33,3 \%$ de los pacientes con diagnostico de meningitis falleció, lo cual es compatible con el 30-35\% de mortalidad esperada en pacientes con criptococosis meníngea que reciben tratamiento con $\mathrm{AmB}$ deoxicolato según la literatura(Burke, 2008 ; Reese \& Betts, 2004).

Estos resultados nos estimulan a incorporar en un trabajo próximo el total de pacientes tratados con anfotericina $\mathrm{B}$, tanto niños como adultos y con distintas infecciones fúngicas.

\section{REFERENCIAS}

Bagnis,C.I. \& Deray ， G.(2002). Amphotericin B nephrotoxicity . Saudi. J Kidney Dis. Transplant.13:481-91

Berdichevski, R.; Billodre, L. L.; Crestana, L.; Ceratti, Manfr o, R. (2006). Amphotericin B- related nephrotoxicity in low-risk patients. Braz J Infect Dis.;10:94-9.

Burke, A. C. (2008). Antibiotic Essentials. Seventh edition. Jones and Bartlett.

Castellá, G .; Abar ca, M. L. \& Cabañes, F .J. (2008). Criptococosis y animales de compañía. Rev . Iberoam. Micol. 25: S19S24

Cruz, Ch, R. (2004). Comentarios sobre la eficacia y la nefrotoxicida de la Anfotericina B convencional e intralipidica en adultos con infeccion fungica sistemica y compromiso inmune. Boletín Micológico Vol. 19:31-35

Fica, A. (2004). Tratamiento de infecciones fúngicas sistémicas. III parte: Anfotericina B, aspectos farmacoeconómicos y decisiones terapéuticas. Rev . Chil. Infect. 21:317-326

Flór ez,J.; Armijo,J.A.; Mediavilla,A. (2003). Farmacología humana. Editorial Masson.pp.1173-1179

Gilber t,D.; Moellering,R.; Eliopoulos,G ； Chambers,H.; Saag,M. (2009). The Sanford Guide To Antimicrobial Therapy . Thirty ninth edition. pp. 97-111

Hardman,J.G .; Limbird, L.E.; Goodman ,A.l. （2003). Bases Farmacológicas de la Terapéutica. Editorial Panamericana. Vol II. 49:1247-1255

Laniad o-Laborín, R. \& Cabrales-V argas, M. (2009). Amphotericin B: side ef fects and toxicity Rev . Iberoam. Micol. 26:223-227

Luke, R.G.; Boyle, J.A. (1998). Renal ef fects of amphotericin B lipid complex. Am. J. Kidney Dis. 31:780-785

Mandell, L.; Bennett, J. E. \& Dolin, R. ～(2002). Enfermedades 
infecciosas: principios y práctica. Editorial Panamericana. 253: $3275-3286$

Nucci, M.; Lour eiro,M; Silveira,F .; Casali,AR.; Bouzas,L.F .; Velazco,E. et al. (1999). Comparaison of toxixity of in amphotericin B dextrose with that of amphotericin B in fat emulsion in randomized trial with cancer patients. Antimicrobial agents and chemotheraphy $\quad .43: 1197-1201$

Quinter o,E.; Castañeda,E.; Ruiz,

A. (2005). Distribución ambiental de Cryptococcus neoformans en el departamento de Cundinamarca-Colombia Rev . Iberoam. Micol. 22:93-98
Reese, R.E. \& Betts, R.S. (2004).Enfermedades Infecciosas. Editorial MARBAN. 17:651-654

Sepúlveda,C. \& Afani, A. (2002). SIDA. Editorial Mediterraneo. 12:206-230

Thompson, L. (1997). Anfotericina B: nuevas formas farmaceuticas. Rev .Chil. Infect. 14:258-262

Walsh,T .H.;Finberg,R.\&Arndt,C. (1999).Liposomal amphotericin B for empirical therapy in patients with persistent fever and neutropenia. New England Journal of Medicine. 340:764-771 